\title{
Solving two-dimensional fuzzy Fredholm integral equations via sinc collocation method
}

\author{
Yanying $\mathrm{Ma}^{1 *}\left(\mathrm{D}, \mathrm{Hu} \mathrm{Li}^{2}\right.$ and Suping Zhang ${ }^{1}$
}

\section{"Correspondence:}

ma_yan_ying@126.com

${ }^{1}$ School of Mathematics and

Physics, Anhui Jianzhu University,

Hefei, P.R. China

Full list of author information is

available at the end of the article

\section{Springer}

\begin{abstract}
In this paper, we present a numerical method to solve two-dimensional fuzzy Fredholm integral equations (2D-FFIE) by combing the sinc method with double exponential (DE) transformation. Using this method, the fuzzy Fredholm integral equations are converted into dual fuzzy linear systems. Convergence analysis is performed in terms of the modulus of continuity. Numerical experiments demonstrate the efficiency of the proposed method.
\end{abstract}

Keywords: Fuzzy Fredholm integral equations; Sinc method; DE transformation; Modulus of continuity

\section{Introduction}

Integral equations have some applications in different fields, such as physics, biological models, and so on [1-5]. They can be solved by different numerical or analytical methods. Specifically, fuzzy integral equations also appear in different problems, for example, optimal control theory, mathematical economics, and engineering. The existence and uniqueness of solutions of fuzzy Fredholm integral equations have been studied, which were implemented by means of the Banach fixed point theorem [6-9]. In recent years, the researches on fuzzy differential equations and fuzzy integral equations from both theoretical and numerical points of view have been developed. There were some numerical methods to solve fuzzy differential equations [10-15] and fuzzy integral equations [1623]. Rivaz et al. [24] and Ezzati et al. [25] presented the homotopy perturbation method and fuzzy bivariate Bernestein polynomials method to solve 2D-FFIE, respectively. Other noticeable methods applied to 2D-FFIE were the block-pulse functions [26], triangular functions method [27], cubature method [28], and iterative method [29, 30].

In [22], the sinc method was proposed to solve one-dimensional fuzzy integral equations. However, the convergence analysis was not given. The primary aim of this paper is to extend the application of the sinc method together with DE transformation to find the approximate solution of 2D-FFIE. In addition, we prove the convergence of the proposed method. The proposed method has its advantages such as simple structure, easy to pro-

(c) The Author(s) 2020. This article is licensed under a Creative Commons Attribution 4.0 International License, which permits use, sharing, adaptation, distribution and reproduction in any medium or format, as long as you give appropriate credit to the original author(s) and the source, provide a link to the Creative Commons licence, and indicate if changes were made. The images or other third party material in this article are included in the article's Creative Commons licence, unless indicated otherwise in a credit line to the material. If material is not included in the article's Creative Commons licence and your intended use is not permitted by statutory regulation or exceeds the permitted use, you will need to obtain permission directly from the copyright holder. To view a copy of this licence, visit http://creativecommons.org/licenses/by/4.0/. 
gramming, and high accuracy. In addition, the proposed method does not need iterative operation, and the calculation cost is reduced

The outline of this paper is as follows. In Sect. 2, we introduce some preliminaries and basic definitions. In Sect. 3, we present the proposed algorithm. Section 4 is focused on discussing the convergence of the proposed method. To demonstrate the effectiveness of the proposed method, we will show some numerical results on several tests in Sect. 5 . Finally, some conclusion remarks are given in Sect. 6.

\section{Preliminaries}

\subsection{The basic concepts of fuzzy equations}

First, we review several necessary basic definitions and relevant results about fuzzy numbers and fuzzy-number-valued functions.

Definition 2.1 (see [31]) A fuzzy number is a function $u: \mathbb{R} \rightarrow[0,1]$ satisfying the following properties:

(1) $u$ is upper semicontinuous over $\mathbb{R}$,

(2) $u(x)=0$ outside of some interval $[a, b] \subset \mathbb{R}$,

(3) there exist real numbers $a$ and $b$ with $c \leq a \leq b \leq d$, such that $u$ is monotonic increasing on $[c, a]$, and monotonic decreasing on $[b, d]$, and $u(x)=1$ for all $x \in[a, b]$,

(4) $u$ is a fuzzy convex set, that is, for all $x, y \in \mathbb{R}$ and $\lambda \in[0,1]$, $u(\lambda x+(1-\lambda) y) \geq \min \{u(x), u(y)\}$.

The set of all fuzzy numbers is denoted by $\mathbb{R}_{\digamma}$. Note that every $\alpha \in \mathbb{R}$ can be seen as a fuzzy number $\alpha=\chi_{\{\alpha\}}$, and so $\mathbb{R} \subset \mathbb{R}_{\digamma}$.

Based on [32], the fuzzy number $r \in[0,1]$ in parametric form is denoted by an ordered pair of functions $(\underline{u}(r), \bar{u}(r))$, which satisfies the following requirements:

(1) $\underline{u}(r)$ is a bounded left-continuous nondecreasing function on $[0,1]$,

(2) $\bar{u}(r)$ is a bounded left-continuous nonincreasing function on $[0,1]$,

(3) $\underline{u}(r) \leq \bar{u}(r)$.

It is generally known that the addition and multiplication operations of real numbers can be extended to fuzzy numbers. For all $u=(\underline{u}(r), \bar{u}(r)), v=(\underline{v}(r), \bar{v}(r))$, and $k \in \mathbb{R}$, we have:

(1) $u=v$ if and only if $\underline{u}(r)=\underline{v}(r)$ and $\bar{u}(r)=\bar{v}(r)$,

(2) $u \oplus v=(\underline{u}(r)+\underline{v}(r), \bar{u}(r)+\bar{v}(r))$,

(3)

$$
k \otimes u= \begin{cases}(k \underline{u}(r), k \bar{u}(r)), & k \geq 0, \\ (k \bar{u}(r), k \underline{u}(r)), & k<0 .\end{cases}
$$

Definition 2.2 (see [33]) For all $u=(\underline{u}(r), \bar{u}(r))$ and $v=(\underline{v}(r), \bar{v}(r))$, the quantity $D(u, v)=$ $\sup _{r \in[0,1]} \max \{|\underline{u}(r)-\underline{v}(r)|,|\bar{u}(r)-\bar{v}(r)|\}$ is the distance between $u$ and $v$.

It is proved that $\left(\mathbb{R}_{\digamma}, D\right)$ is a complete metric space, which possesses the following properties:

(1) $D(u \oplus w, v \oplus w)=D(u, v) \forall u, v, w \in \mathbb{R}_{\digamma}$,

(2) $D(k \otimes u, k \otimes v)=|k| D(u, v) \forall u, v \in \mathbb{R}_{\digamma}, k \in \mathbb{R}$,

(3) $D(u \oplus v, w \oplus e) \leq D(u, w)+D(v, e) \forall u, v, w, e \in \mathbb{R}_{\digamma}$. 
Definition 2.3 (see [34]) Suppose $f, g:[a, b] \rightarrow \mathbb{R}_{\digamma}$ are fuzzy real-valued functions. The uniform distance between $f, g$ is defined by $D_{U}=\sup \{D(f(x), g(x)) \mid x \in[a, b]\}$.

Definition 2.4 (see [35]) If for every $\varepsilon>0$, there exists $\delta>0$ such that $D\left(f(x), f\left(x_{0}\right)\right)<\varepsilon$ whenever $x \in[a, b],\left|x-x_{0}\right|<\delta$, then the fuzzy real-valued function $f:[a, b] \rightarrow \mathbb{R}_{\digamma}$ is referred to as continuous at the point $x_{0} \in[a, b]$. If $f$ is continuous at each $x_{0} \in[a, b]$, then $f$ is called a fuzzy continuous function.

In addition, $C_{\digamma}([a, b] \times[c, d])$ represents the space of all fuzzy continuous functions $f:[a, b] \times[c, d] \rightarrow \mathbb{R}_{\digamma}$.

Definition 2.5 (see [29]) Suppose $f:[a, b] \times[c, d] \rightarrow \mathbb{R}_{\digamma}$ is a bounded mapping. Then the function $\omega_{[a, b] \times[c, d]}(f, \cdot): \mathbb{R}_{+} \cup 0 \rightarrow \mathbb{R}_{+}$is defined by $\omega_{[a, b] \times[c, d]}(f, \delta)=\sup \{D(f(x, y), f(s, t))$ : $\left.x, s \in[a, b] ; y, t \in[c, d] ; \sqrt{(x-s)^{2}+(y-t)^{2}} \leq \delta\right\}$ is referred as to the modulus of oscillation of $f$ on $[a, b] \times[c, d]$.

Definition 2.6 (see [29]) Suppose that $f:[a, b] \times[c, d] \rightarrow \mathbb{R}_{\digamma}$, and $\Delta_{x}^{n}: a=x_{0}<x_{1}<$ $\cdots<x_{n}=b$ and $\Delta_{y}^{n}: c=y_{0}<y_{1}<\cdots<y_{n}=d$ are two segmentations of the intervals $[a, b]$ and $[c, d]$, respectively. Consider any intermediate points $\xi_{i} \in\left[x_{i-1}, x_{i}\right], \eta_{j} \in\left[y_{j-1}, y_{j}\right](i, j=$ $1, \ldots, n)$ and $\delta:[a, b] \rightarrow \mathbb{R}_{\digamma}, \sigma:[c, d] \rightarrow \mathbb{R}_{\digamma}$. For simplify, the divisions $P_{x}=\left(\left[x_{i-1}, x_{i}\right] ; \xi_{i}\right)$ and $P_{y}=\left(\left[y_{j-1}, y_{j}\right] ; \eta_{j}\right)(i, j=1, \ldots, n)$ are abbreviated to $P_{x}=\left(\Delta^{n}, \xi\right)$ and $P_{y}=\left(\Delta^{n}, \eta\right)$, which are called as $\delta$-fine and $\sigma$-fine, respectively, if $\left[x_{i-1}, x_{i}\right] \subset\left(\xi_{i}-\delta\left(\xi_{i}\right), \xi_{i}+\delta\left(\xi_{i}\right)\right)$ and $\left[y_{j-1}, y_{j}\right] \subset\left(\eta_{j}-\sigma\left(\eta_{j}\right), \eta_{j}+\sigma\left(\eta_{j}\right)\right)$.

The function $f$ is said to be two-dimensional Henstock integrable to $I \in \mathbb{R}_{\digamma}$ if for each $\varepsilon>0$, there are functions $\delta:[a, b] \rightarrow \mathbb{R}_{+}$and $\sigma:[c, d] \rightarrow \mathbb{R}_{+}$such that for any $\delta$-fine and $\sigma$ fine divisions, we have $D\left(\sum_{i=0}^{n} \sum_{j=0}^{n}\left(x_{i}-x_{i-1}\right)\left(y_{j}-y_{j-1}\right) \otimes f\left(\xi_{i}, \eta_{j}\right), I\right)<\varepsilon$, where $\sum$ denotes the fuzzy summation. Then $I$ is called the two-dimensional Henstock integral of $f$ and denoted by $I(f)=(F H) \int_{c}^{d}(F H) \int_{a}^{b} f(x, y) d x d y$. If the functions $\delta$ and $\sigma$ are constant, this integral is called the Riemann integral. In this case, $I \in \mathbb{R}_{\digamma}$ is known as the two-dimensional integral of $f$ on $[a, b] \times[c, d]$ and is denoted by $(F R) \int_{c}^{d}(F R) \int_{a}^{b} f(x, y) d x d y$.

Lemma 2.1 If $f$ and $g$ are Henstock-integrable mappings on $[a, b] \times[c, d]$ and $D(f(x, y)$, $g(x, y))$ is Lebesgue integrable, then

$$
\begin{aligned}
& D\left((F H) \int_{c}^{d}(F H) \int_{a}^{b} f(x, y) d x d y,(F H) \int_{c}^{d}(F H) \int_{a}^{b} g(x, y) d x d y\right) \\
& \quad \leq(L) \int_{c}^{d}(L) \int_{a}^{b} D(f(x, y), g(x, y)) d x d y .
\end{aligned}
$$

Definition 2.7 (see [36]) The fuzzy linear system

$$
\left\{\begin{array}{l}
\sum_{j=1}^{m} a_{1 j} \otimes x_{j}=\sum_{j=1}^{m} b_{1 j} \otimes x_{j} \oplus y_{1}, \\
\sum_{j=1}^{m} a_{2 j} \otimes x_{j}=\sum_{j=1}^{m} b_{2 j} \otimes x_{j} \oplus y_{2}, \\
\vdots \\
\sum_{j=1}^{m} a_{m j} \otimes x_{j}=\sum_{j=1}^{m} b_{m j} \otimes x_{j} \oplus y_{m},
\end{array}\right.
$$


where $A=\left(a_{i j}\right)_{m \times m}$ and $B=\left(b_{i j}\right)_{m \times m}$ are crisp coefficient matrices, and $Y=\left(y_{1}, \ldots, y_{m}\right)^{T}$ is a fuzzy vector, is called a dual fuzzy linear system, and $X=\left(x_{1}, \ldots, x_{m}\right)^{T}$ is called a solution of the fuzzy linear system (2).

Definition 2.8 (see [36]) A fuzzy number vector $\left(x_{1}, x_{2}, \ldots, x_{m}\right)^{T}$ given by $x_{j}=\left(\underline{x}_{j}(r), \bar{x}_{j}(r)\right)$ $(j=1, \ldots, m, 0 \leq r \leq 1)$ is called a solution of Eq. (2) if

$$
\begin{aligned}
& \overline{\sum_{j=1}^{m} a_{i j} x_{j}}=\sum_{j=1}^{m} \underline{a_{i j} x_{j}}=\sum_{j=1}^{m} \underline{b_{i j} x_{j}}+y_{i}, \\
& \overline{\sum_{j=1}^{m} a_{i j} x_{j}}=\sum_{j=1}^{m} \overline{a_{i j} x_{j}}=\sum_{j=1}^{m} \overline{b_{i j} x_{j}}+y_{i} \quad(i=1, \ldots, m) .
\end{aligned}
$$

Next, we introduce the method of [36] for solving Eq. (2). To solve Eq. (2), we write it in the form

$$
(S-T) X=Y,
$$

where the elements of $S=\left(s_{i j}\right)_{2 m \times 2 m}$ and $T=\left(t_{i j}\right)_{2 m \times 2 m}$ are determined as follows:

$$
\begin{aligned}
& a_{i j} \geq 0 \quad \Longrightarrow \quad s_{i j}=a_{i j}, \quad s_{i+m, j+m}=a_{i j}, \\
& a_{i j}<0 \quad \Longrightarrow \quad s_{i, j+m}=-a_{i j}, \quad s_{i+m, j}=-a_{i j} \text {, } \\
& b_{i j} \geq 0 \quad \Longrightarrow \quad t_{i j}=b_{i j}, \quad t_{i+m, j+m}=b_{i j} \text {, } \\
& b_{i j}<0 \quad \Longrightarrow \quad t_{i, j+m}=-b_{i j}, \quad t_{i+m, j}=-b_{i j} \text {, }
\end{aligned}
$$

assuming that the remaining $s_{i j}$ and $t_{i j}$ are zeros. Moreover, the right-hand side vector and the unknowns are

$$
\begin{aligned}
& Y=\left(\underline{y}_{1}, \underline{y}_{2}, \ldots, \underline{y}_{m},-\bar{y}_{1},-\bar{y}_{2}, \ldots,-\bar{y}_{m}\right)^{T}, \\
& X=\left(\underline{x}_{1}, \underline{x}_{2}, \ldots, \underline{x}_{m},-\bar{x}_{1},-\bar{x}_{2}, \ldots,-\bar{x}_{m}\right)^{T},
\end{aligned}
$$

respectively. The structures of $S$ and $T$ suggest that $s_{i j} \geq 0, t_{i j} \geq 0(i, j=1, \ldots, 2 m)$ and

$$
S=\left[\begin{array}{ll}
B_{1} & C_{1} \\
C_{1} & B_{1}
\end{array}\right], \quad T=\left[\begin{array}{ll}
B_{2} & C_{2} \\
C_{2} & B_{2}
\end{array}\right],
$$

where $B_{1}$ and $B_{2}$ include the positive entries of $A$ and $B$, respectively, and $C_{1}$ and $C_{2}$ include the absolute values of negative entries of $A$ and $B$, respectively. It is clear that

$$
A=B_{1}-C_{1}, \quad B=B_{2}-C_{2} .
$$

\subsection{Sinc method}

Definition 2.9 (see [37]) Let $f$ be a function on $\mathbb{R}$ with step size $h>0$. Its Whittaker cardinal is defined by the series

$$
C(f, h)(x)=\sum_{-\infty}^{\infty} f(i h) S(i, h)(x)
$$


whenever this series converges; $S(i, h)(x)=\frac{\sin [\pi(x / h-i)]}{\pi(x / h-i)}$ is known as the $j$ th sinc function.

Definition 2.10 (see [37]) A function $g(t)$ is said to decay double exponentially if there exist constants $\alpha$ and $C$ such that

$$
|g(t)| \leq C \exp (-\alpha \exp |t|), \quad t \in(-\infty, \infty)
$$

Equivalently, a function $g(t)$ is said to decay double exponentially with respect to conformal map $\phi$ if there exist constants $\alpha$ and $C$ such that

$$
\left|g(\phi(t)) \phi^{\prime}(t)\right| \leq C \exp (-\alpha \exp |t|), \quad t \in(-\infty, \infty)
$$

We describe the following sinc quadrature rule by means of DE transformation, which has been fully discussed in [38]:

$$
\int_{a}^{b} f(x) d x=h \sum_{i=-N}^{N} f(\phi(i h)) \phi^{\prime}(i h)+O\left(\exp \left(\frac{-2 \pi d N}{\log (2 \pi d N / \alpha)}\right)\right)
$$

where $h=\frac{\log (2 \pi d N / \alpha)}{N}$ and

$$
\begin{aligned}
& \phi(t)=\frac{b-a}{2} \tanh \left(\frac{\pi}{2} \sinh (t)\right)+\frac{b+a}{2}, \\
& \phi^{\prime}(t)=\frac{b-a}{2} \frac{\pi / 2 \cosh (t)}{\cosh ^{2}(\pi / 2 \sinh (t))}, \quad t \in \mathbb{R} .
\end{aligned}
$$

By [39], if $f(x, y)$ is decaying double exponentially with respect to conformal maps $\phi_{1}(s)=$ $\frac{b-a}{2} \tanh \left(\frac{\pi}{2} \sinh (s)\right)+\frac{b+a}{2}$ and $\phi_{2}(t)=\frac{d-c}{2} \tanh \left(\frac{\pi}{2} \sinh (t)\right)+\frac{d+c}{2}$, then the function $f(x, y)$ can be expanded in series of sinc function as follows:

$$
\begin{aligned}
f\left(\phi_{1}(t), \phi_{2}(s)\right) \phi_{1}^{\prime}(s) \phi_{2}^{\prime}(t)= & \sum_{i=-\infty}^{\infty} \sum_{j=-\infty}^{\infty} f\left(\phi_{1}\left(i h_{1}\right), \phi_{2}\left(j h_{2}\right)\right) \phi_{1}^{\prime}\left(i h_{1}\right), \phi_{2}^{\prime}\left(j h_{2}\right) \\
& \times \operatorname{sinc}\left(\frac{t}{h_{1}}-i\right) \operatorname{sinc}\left(\frac{s}{h_{2}}-j\right)+e\left(t, s, h_{1}, h_{2}\right),
\end{aligned}
$$

where $e\left(s, t, h_{1}, h_{2}\right)$ is the remainder term, which depends on the variables $s, t$ and the mesh sizes $h_{1}=\frac{\log \left(2 \pi d_{1} N / \alpha_{1}\right)}{N}$ and $h_{2}=\frac{\log \left(2 \pi d_{2} M / \alpha_{2}\right)}{M}$. Integrating this expression with respect to $x$ and $y$, we derive

$$
\int_{a}^{b} \int_{c}^{d} f(x, y) d x d y=\int_{-\infty}^{\infty} \int_{-\infty}^{\infty} f\left(\phi_{1}(s), \phi_{2}(t)\right) \phi_{1}^{\prime}(s) \phi_{2}^{\prime}(t) d s d t .
$$

Using the termwise integration, it follows that

$$
\begin{aligned}
\int_{a}^{b} \int_{c}^{d} f(x, y) d x d y= & h_{1} h_{2} \sum_{i=-N}^{N} \sum_{i=-M}^{M} f\left(\phi_{1}\left(i h_{1}\right), \phi_{2}\left(j h_{2}\right)\right) \phi_{1}^{\prime}\left(i h_{1}\right) \phi_{2}^{\prime}\left(j h_{2}\right) \\
& +O\left(\exp \left(\frac{-2 \pi d_{1}}{h_{1}}\right)\right)+O\left(\exp \left(\frac{-2 \pi d_{2}}{h_{2}}\right)\right) .
\end{aligned}
$$




\section{The proposed approach}

We consider the following 2D-FFIE:

$$
u(x, y)=f(x, y) \oplus \lambda \int_{a}^{b} \int_{c}^{d} K(x, y, s, t) \otimes u(s, t) d s d t, \quad(x, y) \in E,
$$

where $\lambda>0, E=[a, b] \times[c, d], K$ is crisp given function on $E \times E$, and $u(x, y), f(x, y)$ are continuous fuzzy real-valued functions.

Here we present a numerical method to solve Eq. (10). Applying the quadrature formula (9) to the integral term of Eq. (10), we obtain

$$
\begin{aligned}
& \int_{a}^{b} \int_{c}^{d} K(x, y, s, t) \otimes u(s, t) d s d t \\
& \quad \approx h_{1} h_{2} \sum_{i=-N}^{N} \sum_{j=-M}^{M} K\left(x, y, \phi_{1}\left(i h_{1}\right), \phi_{2}\left(j h_{2}\right)\right) \phi_{1}^{\prime}\left(i h_{1}\right) \phi_{2}^{\prime}\left(j h_{2}\right) \otimes u_{i j},
\end{aligned}
$$

where $u_{i j}$ is an approximation value of $u\left(x_{i}, y_{j}\right)$ with $x_{i}=\phi_{1}\left(i h_{1}\right)$ and $y_{j}=\phi_{2}\left(j h_{2}\right)$. By substituting Eq. (11) into Eq. (10) the latter can be written as

$$
u(x, y)=f(x, y) \oplus \lambda h_{1} h_{2} \sum_{i=-N}^{N} \sum_{j=-M}^{M} K\left(x, y, \phi_{1}\left(i h_{1}\right), \phi_{2}\left(j h_{2}\right)\right) \phi_{1}^{\prime}\left(i h_{1}\right) \phi_{2}^{\prime}\left(j h_{2}\right) \otimes u_{i j} .
$$

To determine the unknown values $u_{i j}(i=-N, \ldots, N ; j=-M, \ldots, M)$, choosing the sinc points $x_{k}=\phi_{1}\left(k h_{1}\right)$ and $y_{l}=\phi_{2}\left(l h_{2}\right)$ as collocation points, we get

$$
u\left(x_{k}, y_{l}\right)=f\left(x_{k}, y_{l}\right) \oplus \lambda h_{1} h_{2} \sum_{i=-N}^{N} \sum_{j=-M}^{M} K\left(x_{k}, y_{l}, \phi_{1}\left(i h_{1}\right), \phi_{2}\left(j h_{2}\right)\right) \phi_{1}^{\prime}\left(i h_{1}\right) \phi_{2}^{\prime}\left(j h_{2}\right) \otimes u_{i j}
$$

Equivalently, the dual fuzzy linear systems can be shown as follows:

$$
A \otimes U=B \otimes U+F,
$$

where $A=\left(a_{i j}^{k l}\right)$ and $B=\left(b_{i j}^{k l}\right)(i, k=-N, \ldots, N ; j, l=-M, \ldots, M)$ are $m \times m(m=(2 N+1) \times$ $(2 M+1))$ fuzzy matrices. Also, $F$ and $U$ are $m \times 1$ fuzzy vectors, and

$$
\begin{aligned}
& A=I_{m \times m}, \quad b_{i j}^{k l}=\lambda h_{1} h_{2} K\left(x_{k}, y_{l}, \phi_{1}\left(i h_{1}\right), \phi_{2}\left(j h_{2}\right)\right), \\
& F=\left[f\left(x_{-N}, y_{-M}\right), \ldots, f\left(x_{-N}, y_{M}\right), \ldots, f\left(x_{N}, y_{-M}\right), \ldots, f\left(x_{N}, y_{M}\right)\right]^{T}, \\
& U=\left[u_{-N,-M}, \ldots, u_{-N, M}, \ldots, u_{N,-M}, \ldots, u_{N, M}\right]^{T} .
\end{aligned}
$$

By Definition 2.8 the coefficient matrices $A, B$ can be converted to $2 m \times 2 m$ crisp linear systems as follows:

$S \otimes \tilde{U}=\tilde{F} \oplus T \otimes \tilde{U}$, 
where the matrices $S$ and $T$ are defined as in Definition 2.8. Moreover,

$$
\begin{aligned}
& \tilde{F}=\left[\underline{f}\left(x_{-N}, y_{-M}\right), \ldots, f\left(x_{N}, y_{M}\right),-\bar{f}\left(x_{-N}, y_{-M}\right), \ldots,-\bar{f}\left(x_{N}, y_{M}\right)\right]^{T}, \\
& \tilde{U}=\left[\underline{u}_{-N,-M}, \ldots, \underline{u}_{N, M},-\bar{u}_{-N,-M}, \ldots,-\bar{u}_{N, M}\right]^{T} .
\end{aligned}
$$

Equation (16) has a unique solution if and only if the matrix $S-T$ is invertible, and thus

$$
\tilde{U}=(S-T)^{-1} \tilde{F} .
$$

According to the Nyström method in [40], we obtain an approximate solution of Eq. (10) at arbitrary points as follows:

$$
u_{N M}(x, y)=f(x, y) \oplus \lambda h_{1} h_{2} \sum_{i=-N}^{N} \sum_{j=-M}^{M} K\left(x, y, \phi_{1}\left(i h_{1}\right), \phi_{2}\left(j h_{2}\right)\right) \phi_{1}^{\prime}\left(i h_{1}\right) \phi_{2}^{\prime}\left(j h_{2}\right) \otimes u_{i j} .
$$

To simplify the discussion, assuming that $K\left(x, y, \phi_{1}\left(i h_{1}\right), \phi_{2}\left(j h_{2}\right)\right) \phi_{1}^{\prime}\left(i h_{1}\right) \phi_{2}^{\prime}\left(j h_{2}\right) \geq 0$, the approximate solution $u_{N M}(x, y)$ at arbitrary points of Eq. (18) can be written in the form

$$
\begin{aligned}
& \bar{u}_{N M}(x, y)=\bar{f}(x, y) \oplus \lambda h_{1} h_{2} \sum_{i=-N}^{N} \sum_{j=-M}^{M} K\left(x, y, \phi_{1}\left(i h_{1}\right), \phi_{2}\left(j h_{2}\right)\right) \phi_{1}^{\prime}\left(i h_{1}\right) \phi_{2}^{\prime}\left(j h_{2}\right) \otimes \bar{u}_{i j}, \\
& \underline{u}_{N M}(x, y)=\underset{f}{f}(x, y) \oplus \lambda h_{1} h_{2} \sum_{i=-N}^{N} \sum_{j=-M}^{M} K\left(x, y, \phi_{1}\left(i h_{1}\right), \phi_{2}\left(j h_{2}\right)\right) \phi_{1}^{\prime}\left(i h_{1}\right) \phi_{2}^{\prime}\left(j h_{2}\right) \otimes \underline{u}_{i j} \cdot
\end{aligned}
$$

Theorem 3.1 (see [7]) Suppose $A=\left(a_{i j}\right)_{m \times m}$ and $B=\left(B_{i j}\right)_{m \times m}$ are nonnegative matrices. Equation (16) has a unique fuzzy solution if and only if the inverse matrix of $A-B$ exists and has only nonnegative entries.

Remark 1 Based on [7], the matrix $S-T$ is invertible if and only if the matrices $\left(B_{1}+C_{1}\right)$ $\left(B_{2}+C_{2}\right)$ and $\left(B_{1}+C_{2}\right)-\left(B_{2}+C_{1}\right)$ are both invertible. Furthermore, if $(S-T)^{-1}$ exists, then it must have the same structure type as $S-T$, that is,

$$
(S-T)^{-1}=\left[\begin{array}{cc}
E & H \\
H & E
\end{array}\right]
$$

where $E=\frac{1}{2}\left[\left(\left(B_{1}+C_{1}\right)-\left(B_{2}+C_{2}\right)\right)+\left(\left(B_{1}+C_{2}\right)-\left(B_{2}+C_{1}\right)\right)\right]$ and $H=\frac{1}{2}\left[\left(\left(B_{1}+C_{1}\right)-\right.\right.$ $\left.\left.\left(B_{2}+C_{2}\right)\right)-\left(\left(B_{1}+C_{2}\right)-\left(B_{2}+C_{1}\right)\right)\right]$.

\section{Convergence analysis}

In this section, we discuss the convergence of the proposed approach in terms of the modulus of continuity.

Theorem 4.5 Assume that $K(x, y, s, t)$ is analytic and positive on $E \times E$ and that the exact solution $u(x, y)$ is continuous on $E$. If $Q^{*}=\lambda L L_{1}<1$, then we have the following error 
bound:

$$
\begin{aligned}
& D\left(u(x, y), u_{N M}(x, y)\right) \\
& \quad \leq \frac{Q^{*}}{1-Q^{*}}\left(\omega_{[a, b] \times[c, d]}\left(u, \sqrt{\frac{(b-a)^{2} h_{1}^{2}+(d-c)^{2} h_{2}^{2}}{16}}\right)+\frac{(b-a)(d-c)}{L_{0} L_{1}} \varepsilon\right) .
\end{aligned}
$$

Proof Since $K(x, y, s, t)$ is analytic on the compact set of $E \times E$, we obtain that $K(x, y, s, t)$ is uniformly continuous. Therefore for every $\varepsilon>0$, there exists $\delta>0$ such that for all $\left(s_{1}, t_{1}\right),\left(s_{2}, t_{2}\right) \in E$,

$$
\left|K\left(x, y, s_{1}, t_{1}\right)-K\left(x, y, s_{2}, t_{2}\right)\right|<\varepsilon \quad \text { whenever } \sqrt{\left(s_{2}-s_{1}\right)^{2}+\left(t_{2}-t_{1}\right)^{2}}<\delta .
$$

Therefore $K(x, y, s, t)$ is uniformly bounded, that is, there is $L>0$ such that $|K(x, y, s, t)| \leq L$ for all $(x, y, s, t)$. Let $s_{i}=\phi_{1}\left(i h_{1}\right)$ and $t_{j}=\phi_{2}\left(j h_{2}\right)$. According to Eq. (10), Eq. (18) and the properties of $\left(\mathbb{R}_{\digamma}, D\right)$, we have

$$
\begin{aligned}
D\left(u(x, y), u_{N M}(x, y)\right) & \\
= & D\left(f(x, y, f(x, y))+\lambda D\left(\int_{a}^{b} \int_{c}^{d} K(x, y, s, t) \otimes u(s, t) d s d t,\right.\right. \\
& \left.h_{1} h_{2} \sum_{i=-N}^{N} \sum_{j=-M}^{M} K\left(x, y, s_{i}, t_{j}\right) \phi_{1}^{\prime}\left(i h_{1}\right) \phi_{2}^{\prime}\left(j h_{2}\right) \otimes u_{i j}\right) \\
= & \lambda D\left(\int_{a}^{b} \int_{c}^{d} K(x, y, s, t) \otimes u(s, t) d s d t,\right. \\
& \left.h_{1} h_{2} \sum_{i=-N}^{N} \sum_{j=-M}^{M} K\left(x, y, s_{i}, t_{j}\right) \phi_{1}^{\prime}\left(i h_{1}\right) \phi_{2}^{\prime}\left(j h_{2}\right) \otimes u_{i j}\right) \\
\leq & \lambda D\left(\int_{a}^{b} \int_{c}^{d} K(x, y, s, t) \otimes u(s, t) d s d t,\right. \\
& \left.h_{1} h_{2} \sum_{i=-N}^{N} \sum_{j=-M}^{M} K(x, y, s, t) \phi_{1}^{\prime}\left(i h_{1}\right) \phi_{2}^{\prime}\left(j h_{2}\right) \otimes u\left(s_{i}, t_{j}\right)\right) \\
& +\lambda D\left(h_{1} h_{2} \sum_{i=-N}^{N} \sum_{j=-M}^{M} K(x, y, s, t) \phi_{1}^{\prime}\left(i h_{1}\right) \phi_{2}^{\prime}\left(j h_{2}\right) \otimes u\left(s_{i}, t_{j}\right),\right. \\
& \left.h_{1} h_{2} \sum_{i=-N}^{N} \sum_{j=-M}^{M} K\left(x, y, s_{i}, t_{j}\right) \phi_{1}^{\prime}\left(i h_{1}\right) \phi_{2}^{\prime}\left(j h_{2}\right) \otimes u_{i j}\right) \\
& +\lambda D\left(\int_{a}^{b} \int_{c}^{d} u(s, t) d s d t, h_{1} h_{2} \sum_{i=-\infty}^{\infty} \sum_{j=-\infty}^{\infty} \phi_{1}^{\prime}\left(i h_{1}\right) \phi_{2}^{\prime}\left(j h_{2}\right) \otimes u\left(s_{i}, t_{j}\right)\right) \\
& \left.h_{1} h_{2} \sum_{i=-N}^{N} \sum_{j=-M}^{M} K\left(x, y, s_{i}, t_{j}\right) \phi_{1}^{\prime}\left(i h_{1}\right) \phi_{2}^{\prime}\left(j h_{2}\right) \otimes u\left(s_{i}, t_{j}\right)\right) \\
& \sum_{i=-N}^{M} K\left(x, y, s_{i}, t_{j}\right) \phi_{1}^{\prime}\left(i h_{1}\right) \phi_{2}^{\prime}\left(j h_{2}\right) \otimes u\left(s_{i}, t_{j}\right),
\end{aligned}
$$




$$
\begin{aligned}
& +\lambda h_{1} h_{2} \sum_{i=-N}^{N} \sum_{j=-M}^{M}\left|K(x, y, s, t)-K\left(x, y, s_{i}, t_{j}\right)\right| \phi_{1}^{\prime}\left(i h_{1}\right) \phi_{2}^{\prime}\left(j h_{2}\right) D\left(u\left(s_{i}, t_{j}\right), 0\right) \\
& +\lambda h_{1} h_{2} \sum_{i=-N}^{N} \sum_{j=-M}^{M}\left|K\left(x, y, s_{i}, t_{j}\right)\right| \phi_{1}^{\prime}\left(i h_{1}\right) \phi_{2}^{\prime}\left(j h_{2}\right) D\left(u\left(s_{i}, t_{j}\right), u_{i j}\right) \\
& \leq \lambda L D\left(\int_{a}^{b} \int_{c}^{d} u(s, t) d s d t, \sum_{i=-\infty}^{\infty} \sum_{j=-\infty}^{\infty}\left(s_{i+1}-s_{i}\right)\left(t_{j+1}-t_{j}\right) \otimes u\left(s_{i}, t_{j}\right)\right) \\
& +\lambda \varepsilon h_{1} h_{2} \sum_{i=-N}^{N} \sum_{j=-M}^{M} \phi_{1}^{\prime}\left(i h_{1}\right) \phi_{2}^{\prime}\left(j h_{2}\right) D\left(u\left(s_{i}, t_{j}\right), 0\right) \\
& +\lambda L h_{1} h_{2} \sum_{i=-N}^{N} \sum_{j=-M}^{M} \phi_{1}^{\prime}\left(i h_{1}\right) \phi_{2}^{\prime}\left(j h_{2}\right) D\left(u(x, y), u_{N M}(x, y)\right) .
\end{aligned}
$$

Since $u(x, y)$ is continuous, $D\left(u\left(s_{i}, t_{j}\right), 0\right) \leq D(u(x, y), 0) \leq \sup _{(x, y) \in E}\|u(x, y)\|_{\digamma} \leq L_{0}$, and we get

$$
\begin{aligned}
& D\left(u(x, y), u_{N M}(x, y)\right) \\
& \leq \lambda L D\left(\int_{a}^{b} \int_{c}^{d} u(s, t) d s d t, \sum_{i=-\infty}^{\infty} \sum_{j=-\infty}^{\infty}\left(s_{i+1}-s_{i}\right)\left(t_{j+1}-t_{j}\right) \otimes u\left(s_{i}, t_{j}\right)\right) \\
& \quad+\lambda \varepsilon h_{1} h_{2} L_{0} \sum_{i=-\infty}^{\infty} \sum_{j=-\infty}^{\infty} \phi_{1}^{\prime}\left(i h_{1}\right) \phi_{2}^{\prime}\left(j h_{2}\right) \\
& \quad+\lambda L h_{1} h_{2} \sum_{i=-\infty}^{\infty} \sum_{j=-\infty}^{\infty} \phi_{1}^{\prime}\left(i h_{1}\right) \phi_{2}^{\prime}\left(j h_{2}\right) D\left(u(x, y), u_{N M}(x, y)\right) \\
& \leq \lambda L \sum_{i=-\infty}^{\infty} \sum_{j=-\infty}^{\infty}\left(s_{i+1}-s_{i}\right)\left(t_{j+1}-t_{j}\right) \omega_{\left[s_{i}, s_{i+1}\right] \times\left[t_{j}, t_{j+1}\right]}\left(u, \sqrt{\left(s_{i+1}-s_{i}\right)^{2}+\left(t_{j+1}-t_{j}\right)^{2}}\right) \\
& \quad+\lambda \varepsilon L_{0} L_{1}+\lambda L L_{1} D\left(u(x, y), u_{N M}(x, y)\right) .
\end{aligned}
$$

Here, since $h_{1} h_{2} \sum_{i=-\infty}^{\infty} \sum_{j=-\infty}^{\infty} \phi_{1}^{\prime}\left(i h_{1}\right) \phi_{2}^{\prime}\left(j h_{2}\right) \rightarrow(b-a)(d-c)$, there exists a constant $L_{1}>0$ such that $h_{1} h_{2} \sum_{i=-\infty}^{\infty} \sum_{j=-\infty}^{\infty} \phi_{1}^{\prime}\left(i h_{1}\right) \phi_{2}^{\prime}\left(j h_{2}\right) \leq L_{1}$. On the other hand, based on the differential mean value theorem, we have $s_{i+1}-s_{i}=\phi_{1}^{\prime}\left(\xi_{i}\right) h_{1} \leq \frac{b-a}{4} h_{1}$ and $t_{j+1}-t_{j}=\phi_{2}^{\prime}\left(\eta_{j}\right) h_{2} \leq \frac{d-c}{4} h_{2}$, and thus

$$
\begin{aligned}
& D\left(u(x, y), u_{N M}(x, y)\right) \\
& \leq \lambda L \sum_{i=-\infty}^{\infty} \sum_{j=-\infty}^{\infty}\left(s_{i+1}-s_{i}\right)\left(t_{j+1}-t_{j}\right) \omega_{[a, b] \times[c, d]}\left(u, \sqrt{\frac{(b-a)^{2} h_{1}^{2}+(d-c)^{2} h_{2}^{2}}{16}}\right) \\
& \quad+\lambda \varepsilon L_{0} L_{1}+\lambda L L_{1} D\left(u(x, y), u_{N M}(x, y)\right) \\
& \leq \lambda L(b-a)(d-c) \omega_{[a, b] \times[c, d]}\left(u, \sqrt{\frac{(b-a)^{2} h_{1}^{2}+(d-c)^{2} h_{2}^{2}}{16}}\right) \\
& \quad+\lambda \varepsilon L_{0} L_{1}+\lambda L L_{1} D\left(u(x, y), u_{N M}(x, y)\right) .
\end{aligned}
$$


Therefore we can conclude that

$$
\begin{aligned}
D\left(u(x, y), u_{N M}(x, y)\right) \leq & \frac{\lambda L(b-a)(d-c)}{1-\lambda L L_{1}} \omega_{[a, b] \times[c, d]}\left(u, \sqrt{\frac{(b-a)^{2} h_{1}^{2}+(d-c)^{2} h_{2}^{2}}{16}}\right) \\
& +\frac{\lambda L_{0} L_{1}}{1-\lambda L L_{1}} \varepsilon .
\end{aligned}
$$

Thus the proof of the theorem is completed.

Remark 2 Because $Q^{*}<1$, it is easy to verify that $\lim _{\substack{N, M \rightarrow \infty \\ h_{1}, h_{2} \rightarrow 0}} \sup _{x, y \in E} D\left(u(x, y), u_{N M}(x, y)\right)=$ 0 , that is, the proposed algorithm is convergent.

\section{Numerical experiments}

In this section, the performance of our numerical approach was tested on three illustrative examples. All experiments are implemented using MATLAB. Here we choose $M=N$ and $h_{1}=h_{2}=\frac{\log \frac{\pi}{2}}{N}$ in all tables and figures. To simplify the discussion, we use $\underline{E}^{r}=\left|\underline{u}-\underline{u}_{N M}\right|$ and $\bar{E}^{r}=\left|\bar{u}-\bar{u}_{N M}\right|$.

Example 5.1 Consider the following 2D-FFIE in Eq. (10) with

$$
f(x, y, r)=x \sin \left(\frac{y}{2}\right)\left(r^{2}+r\right), \quad \bar{f}(x, y, r)=x \sin \left(\frac{y}{2}\right)\left(4-r^{3}-r\right),
$$

$\lambda=1$, and $K(x, y, s, t)=x^{2} y s$ for $0 \leq x, y, s, t \leq 1$. The exact solution of this example is

$$
\begin{aligned}
& \underline{u}(x, y, r)=\left(x \sin \left(\frac{y}{2}\right)-\frac{16}{21}\left(\cos \left(\frac{1}{2}\right)-1\right) x^{2} y\right)\left(r^{2}+r\right), \\
& \bar{u}(x, y, r)=\left(x \sin \left(\frac{y}{2}\right)-\frac{16}{21}\left(\cos \left(\frac{1}{2}\right)-1\right) x^{2} y\right)\left(4-r^{3}-r\right) .
\end{aligned}
$$

From Table 1 and Fig. 1 we can find that the numerical solutions are more and more close to the exact solutions as $N$ and $M$ increase. In Table 2, comparing the proposed method with the triangular function method [27] and block pulse function method [26], we see that the proposed method has a higher accuracy and much smaller error with less

\begin{tabular}{|c|c|c|c|c|c|c|}
\hline \multirow[t]{2}{*}{$r$} & \multicolumn{2}{|l|}{$N=4$} & \multicolumn{2}{|l|}{$N=8$} & \multicolumn{2}{|l|}{$N=16$} \\
\hline & $\underline{\underline{E}}^{r}$ & $\vec{E}^{\prime}$ & $\underline{\underline{E}}^{r}$ & $\vec{E}^{\prime}$ & $\underline{E}^{r}$ & $\vec{E}^{\prime}$ \\
\hline 0.00 & 0 & $5.2 \mathrm{e}-06$ & 0 & 2.7e-09 & 0 & $6.7 \mathrm{e}-16$ \\
\hline 0.20 & $3.1 \mathrm{e}-07$ & $5.0 \mathrm{e}-06$ & $1.6 \mathrm{e}-10$ & 2.6e-09 & $4.9 e-17$ & $6.7 e-16$ \\
\hline 0.40 & 7.3e-07 & 4.6e-06 & $3.8 \mathrm{e}-10$ & $2.4 \mathrm{e}-09$ & $1.1 \mathrm{e}-16$ & $7.2 \mathrm{e}-16$ \\
\hline 0.60 & $1.3 e-06$ & $4.2 \mathrm{e}-06$ & $6.5 e-10$ & 2.2e-09 & $1.7 \mathrm{e}-16$ & $5.6 e-16$ \\
\hline 0.80 & $1.9 \mathrm{e}-06$ & $3.5 \mathrm{e}-06$ & $9.8 \mathrm{e}-10$ & $1.8 \mathrm{e}-09$ & $2.5 e-16$ & $5.6 e-16$ \\
\hline 1.00 & $2.6 \mathrm{e}-06$ & $2.6 \mathrm{e}-06$ & $1.4 \mathrm{e}-09$ & $1.4 \mathrm{e}-09$ & $3.3 e-16$ & $3.3 e-16$ \\
\hline
\end{tabular}
collocation points. The condition number of the matrix $S-T$ is uniformly bounded with infinity norm in Fig. 2. This shows that the current method is stable. Figure 3 shows that the numerical solutions are in good agreement with exact solution.

Table 1 Numerical errors on the level sets with $N=M$ in $(x, y)=(0.5 .0 .5)$ for Example 5.1 


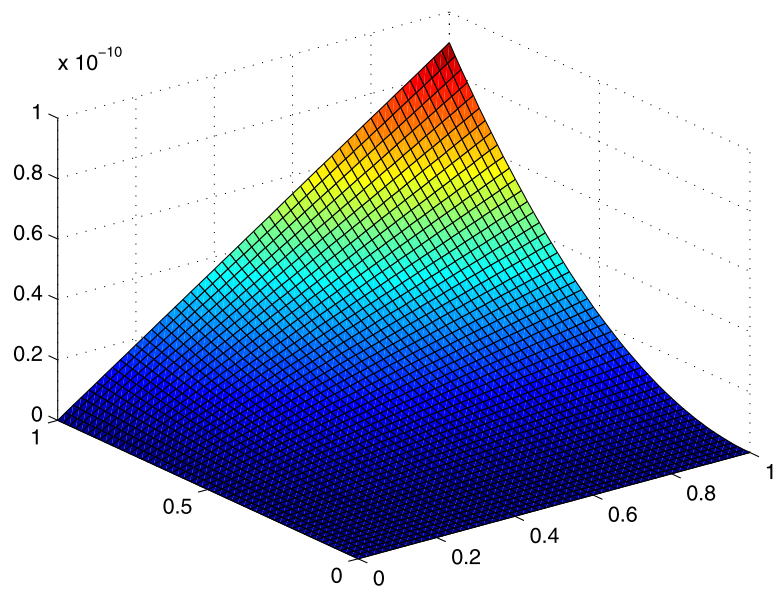

(a) $\underline{e}_{N M}(\mathrm{x}, \mathrm{y}, 0.5)$

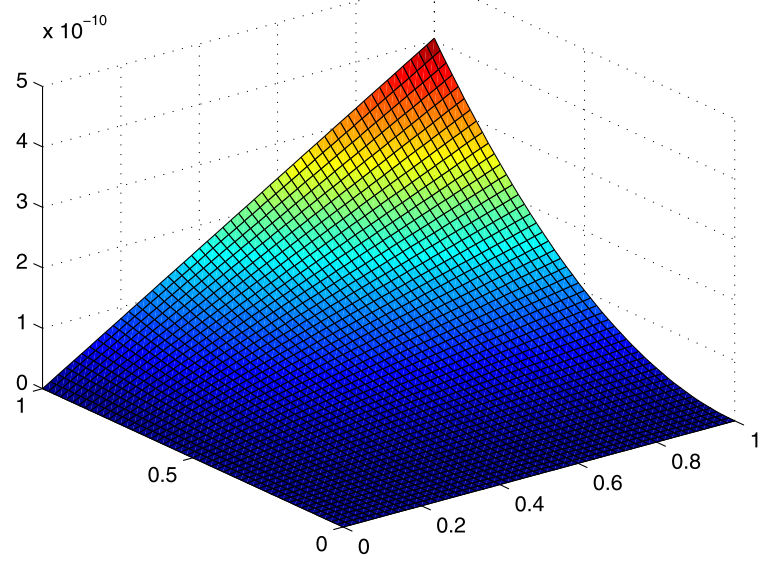

(b) $\bar{e}_{N M}(\mathrm{x}, \mathrm{y}, 0.5)$

Figure 1 Numerical errors with $N=10$ of Example 5.1

Table 2 Comparison of methods with $(x, y)=(0.3 .0 .6)$ of Example 5.1

\begin{tabular}{llll}
\hline$r$ & $\begin{array}{l}\text { Present method } \\
n=2 N+1=17\end{array}$ & $\begin{array}{l}\text { Method of [27] } \\
n=2 N=20 \\
\left(\underline{E}^{r}, \bar{E}^{r}\right)\end{array}$ & $\begin{array}{l}\text { Method of [26] } \\
n=52 \\
\left(\underline{E}^{r}, \bar{E}^{r}\right)\end{array}$ \\
\hline 0.1 & $(3.2 \mathrm{E}-11,1.1 \mathrm{e}-09)$ & $(1.5 \mathrm{e}-07,5.3 \mathrm{e}-06)$ & $(0.0007,0.0004)$ \\
0.3 & $(1.1 \mathrm{e}-10,1.1 \mathrm{e}-09)$ & $(5.2 \mathrm{e}-07,5.0 \mathrm{e}-06)$ & $(0.0002,0.0011)$ \\
0.5 & $(2.2 \mathrm{e}-10,9.9 \mathrm{e}-10)$ & $(1.0 \mathrm{e}-06,4.6 \mathrm{e}-06)$ & $(0.0020,0.0008)$ \\
0.7 & $(3.5 \mathrm{e}-10,8.7 \mathrm{e}-10)$ & $(1.6 \mathrm{e}-06,4.0 \mathrm{e}-06)$ & $(0.0007,0.0003)$ \\
0.9 & $(5.0 \mathrm{e}-10,7.0 \mathrm{e}-10)$ & $(2.3 \mathrm{e}-06,3.2 \mathrm{e}-06)$ & $(0.0011,0.0024)$ \\
\hline
\end{tabular}

Example 5.2 Consider the following 2D-FFIE in Eq. (10):

$$
\underline{f}(x, y, r)=r\left(x y+\frac{1}{676}\left(x^{2}+y^{2}-2\right)\right), \quad \bar{f}(x, y, r)=(2-r)\left(x y+\frac{1}{676}\left(x^{2}+y^{2}-2\right)\right),
$$




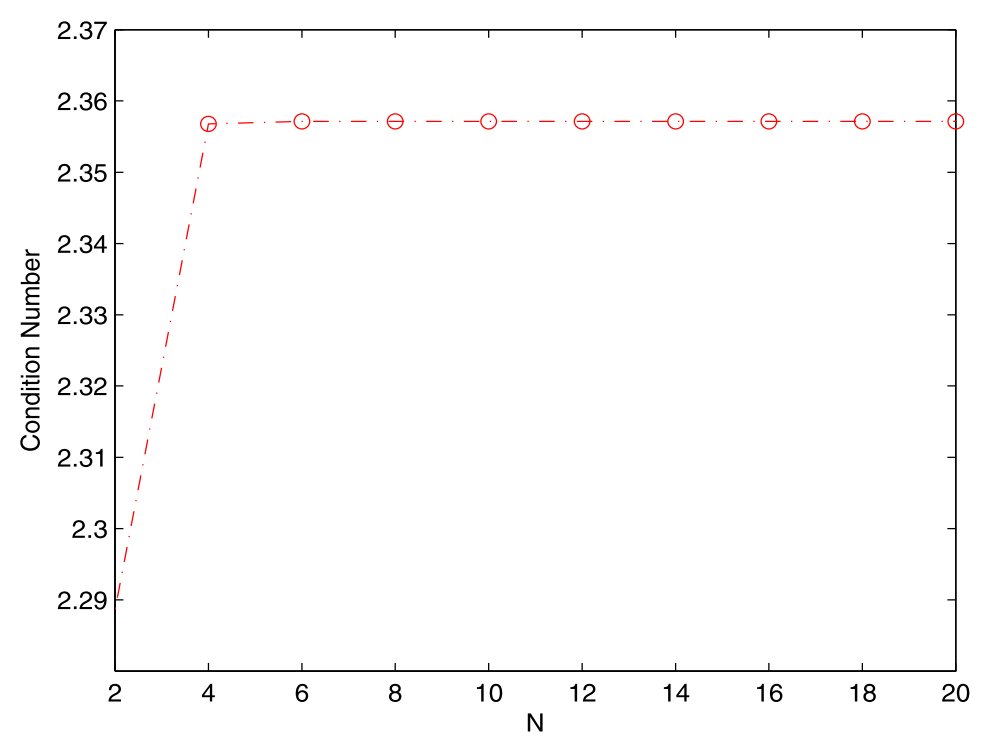

Figure 2 Condition number of the matrix $S-T$ with Example 5.1

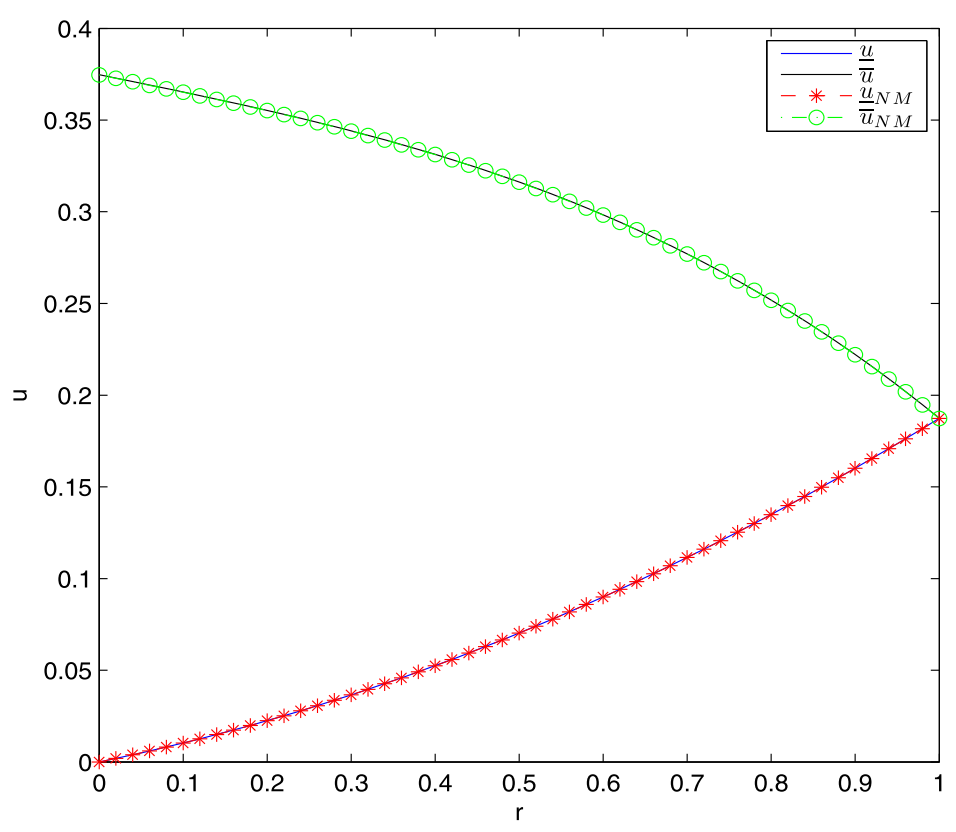

Figure 3 Exact solution and approximate solution with $N=2$ of Example 5.1

$\lambda=1$ and $K(x, y, s, t)=\frac{1}{169}\left(x^{2}+y^{2}-2\right)\left(s^{2}+t^{2}-2\right)$ for $0 \leq x, y, s, t \leq 1$. The exact solution of this example is

$$
\underline{u}(x, y, r)=r x y, \quad \bar{u}(x, y, r)=(2-r) x y .
$$

It is evident from the Table 3 and Fig. 4 that if we increase the collocation points, then the absolute error decreases. Comparing the presented method and the triangular function method [27] in Table 4, we see that the former is more accurate than the latter. Figure 5 
Table 3 Numerical errors on the level sets with $N=M$ in $(x, y)=(0.5 .0 .5)$ for Example 5.2

\begin{tabular}{|c|c|c|c|c|c|c|}
\hline \multirow[t]{2}{*}{ r } & \multicolumn{2}{|l|}{$N=4$} & \multicolumn{2}{|l|}{$N=8$} & \multicolumn{2}{|l|}{$N=16$} \\
\hline & $\underline{E^{r}}$ & $\vec{E}^{\prime}$ & $\underline{E^{r}}$ & $\vec{E}^{\prime}$ & $\underline{\underline{E}}$ & $\vec{E}^{\prime}$ \\
\hline 0.00 & 0 & 7.6e-07 & 0 & $4.3 e-10$ & 0 & $1.1 \mathrm{e}-16$ \\
\hline 0.20 & 7.6e-08 & $6.8 \mathrm{e}-07$ & $4.2 \mathrm{e}-11$ & $3.8 \mathrm{e}-10$ & $1.4 \mathrm{e}-17$ & $5.6 e-17$ \\
\hline 0.40 & $1.5 e-07$ & $6.1 \mathrm{e}-07$ & $8.5 e-11$ & $3.4 \mathrm{e}-10$ & $2.8 \mathrm{e}-17$ & $1.1 \mathrm{e}-16$ \\
\hline 0.60 & $2.3 e-07$ & $5.3 e-07$ & $1.3 e-10$ & $3.0 \mathrm{e}-10$ & $2.8 \mathrm{e}-17$ & $5.6 e-17$ \\
\hline 0.80 & 3.0e-07 & $4.5 e-07$ & $1.7 e-10$ & 2.6e-10 & $5.6 \mathrm{e}-17$ & $5.6 e-17$ \\
\hline 1.00 & $3.8 \mathrm{e}-07$ & $3.9 \mathrm{e}-07$ & $2.1 \mathrm{e}-10$ & $2.1 \mathrm{e}-10$ & $5.6 \mathrm{e}-17$ & $5.6 e-17$ \\
\hline
\end{tabular}

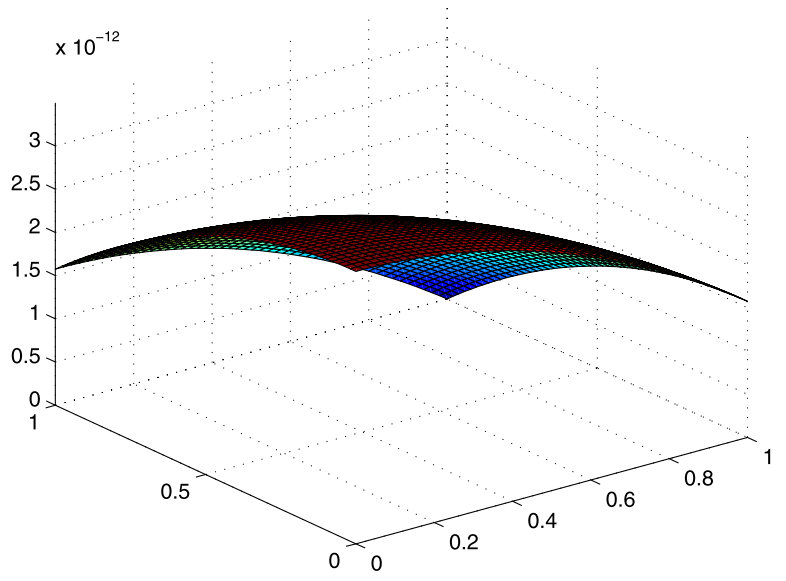

(a) $\underline{e}_{N M}(\mathrm{x}, \mathrm{y}, 0.5)$

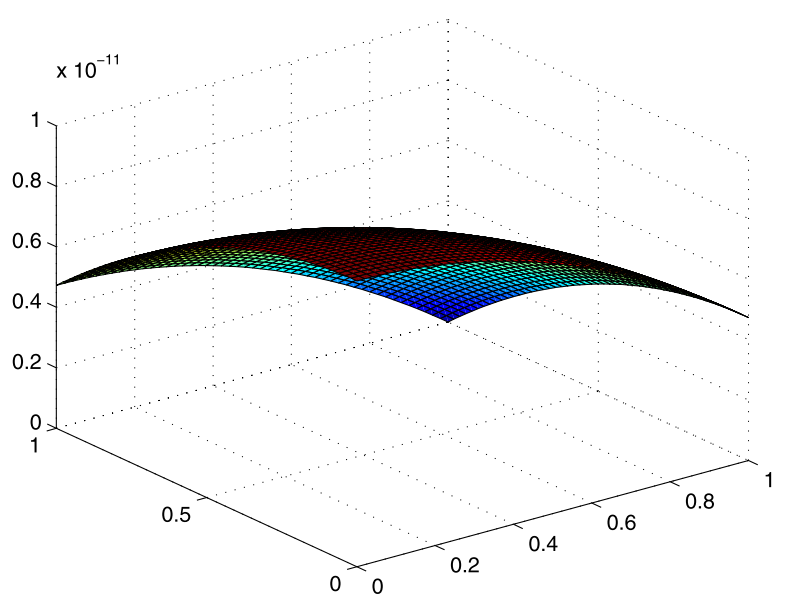

(b) $\bar{e}_{N M}(\mathrm{x}, \mathrm{y}, 0.5)$

Figure 4 Numerical errors with $N=10$ of Example 5.2

suggests that the condition number of the discrete coefficient matrix remains unchanged under different values of $M=N$. In Fig. 6 we plotted the numerical solutions and exact solutions for $M=N=2$. 
Table 4 Comparison of methods with $(x, y)=(0.1 .0 .4)$ of Example 5.2

\begin{tabular}{lll}
\hline$r$ & $\begin{array}{l}\text { Present method } \\
n=2 N+1=17 \\
\left(\underline{E}^{r}, \bar{E}^{r}\right)\end{array}$ & $\begin{array}{l}\text { Method of [27] } \\
n=2 N=24 \\
\left(\underline{E}^{r}, \bar{E}^{r}\right)\end{array}$ \\
\hline 0.1 & $(2.6 \mathrm{e}-11,5.0 \mathrm{e}-10)$ & $(4.9 \mathrm{e}-07,9.4 \mathrm{e}-06)$ \\
0.3 & $(7.8 \mathrm{e}-11,4.4 \mathrm{e}-10)$ & $(1.5 \mathrm{e}-06,8.4 \mathrm{e}-06)$ \\
0.5 & $(1.3 \mathrm{e}-10,3.9 \mathrm{e}-10)$ & $(2.5 \mathrm{e}-06,7.4 \mathrm{e}-06)$ \\
0.7 & $(1.8 \mathrm{e}-10,3.4 \mathrm{e}-10)$ & $(3.4 \mathrm{e}-06,6.4 \mathrm{e}-06)$ \\
0.9 & $(2.3 \mathrm{e}-10,2.9 \mathrm{e}-10)$ & $(4.4 \mathrm{e}-06,5.4 \mathrm{e}-06)$
\end{tabular}

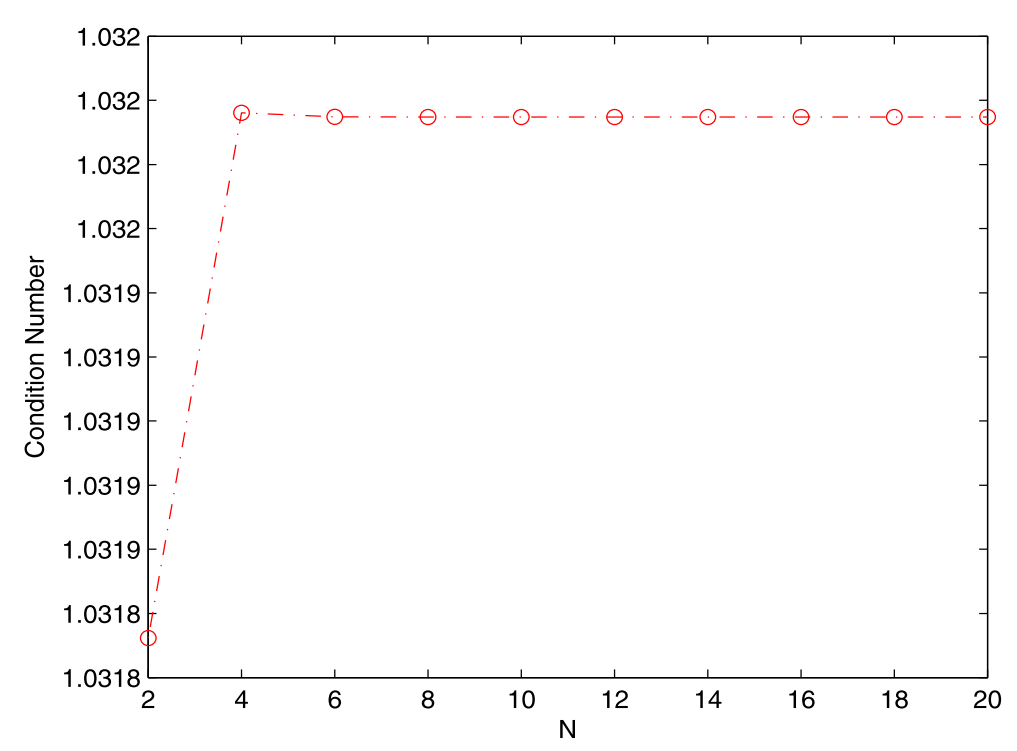

Figure 5 Condition number of the matrix $S-T$ with Example 5.2

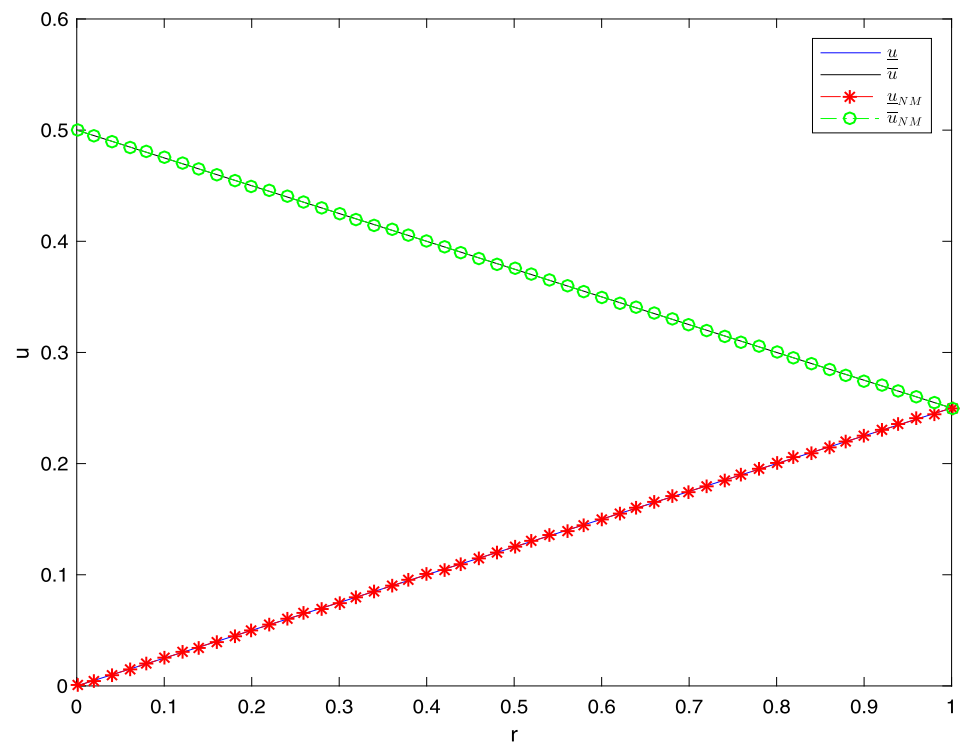

Figure 6 Exact solution and approximate solution with $N=2$ of Example 5.2 
Table 5 Numerical errors on the level sets with $N=M$ in $(x, y)=(0.5 .0 .5)$ for Example 5.3

\begin{tabular}{|c|c|c|c|c|c|c|}
\hline \multirow[t]{2}{*}{$r$} & \multicolumn{2}{|l|}{$N=4$} & \multicolumn{2}{|l|}{$N=8$} & \multicolumn{2}{|l|}{$N=16$} \\
\hline & $\underline{E}^{r}$ & $\vec{E}^{\prime}$ & $\underline{E}^{r}$ & $\vec{E}^{\prime}$ & $\underline{E}^{r}$ & $\vec{E}^{\prime}$ \\
\hline 0.00 & $5.7 \mathrm{e}-05$ & $1.1 \mathrm{e}-04$ & $3.0 \mathrm{e}-08$ & $5.9 \mathrm{e}-08$ & $9.8 e-15$ & $1.9 e-14$ \\
\hline 0.20 & $4.1 e-05$ & $9.7 e-05$ & $2.1 \mathrm{e}-08$ & $5.0 \mathrm{e}-08$ & $6.9 e-15$ & $1.4 \mathrm{e}-14$ \\
\hline 0.40 & $2.0 e-05$ & $8.1 \mathrm{e}-05$ & $1.0 \mathrm{e}-08$ & $4.2 \mathrm{e}-08$ & $3.3 e-15$ & $1.2 \mathrm{e}-14$ \\
\hline 0.60 & $6.0 \mathrm{e}-06$ & $6.8 \mathrm{e}-05$ & $3.1 \mathrm{e}-09$ & $3.5 \mathrm{e}-08$ & $9.2 e-16$ & $8.9 e-15$ \\
\hline 0.80 & $3.3 e-05$ & $6.0 \mathrm{e}-05$ & $1.7 e-08$ & $3.1 \mathrm{e}-08$ & $4.4 \mathrm{e}-15$ & $9.3 e-15$ \\
\hline 1.00 & 5.7e-05 & 5.7e-05 & $3.0 \mathrm{e}-08$ & $3.0 \mathrm{e}-08$ & $9.8 \mathrm{e}-15$ & $9.3 e-15$ \\
\hline
\end{tabular}

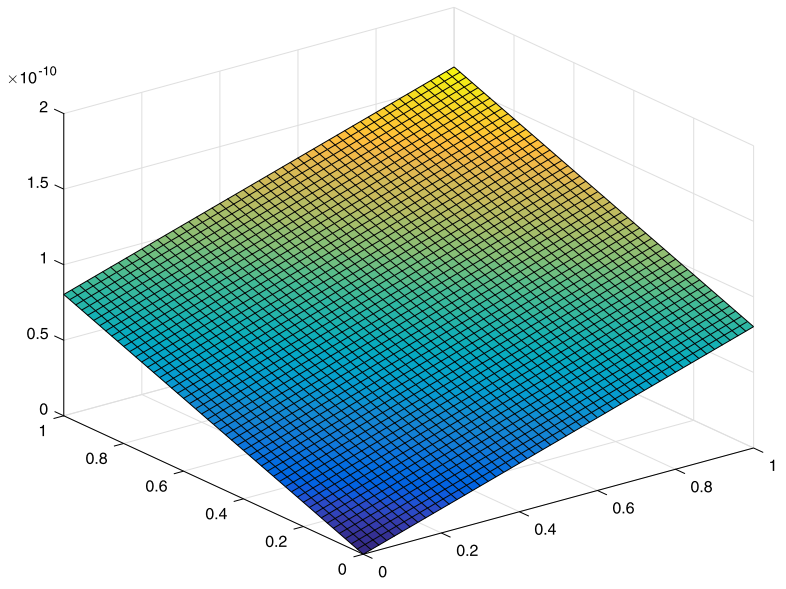

(a) $\underline{e}_{N M}(\mathrm{x}, \mathrm{y}, 0.5)$

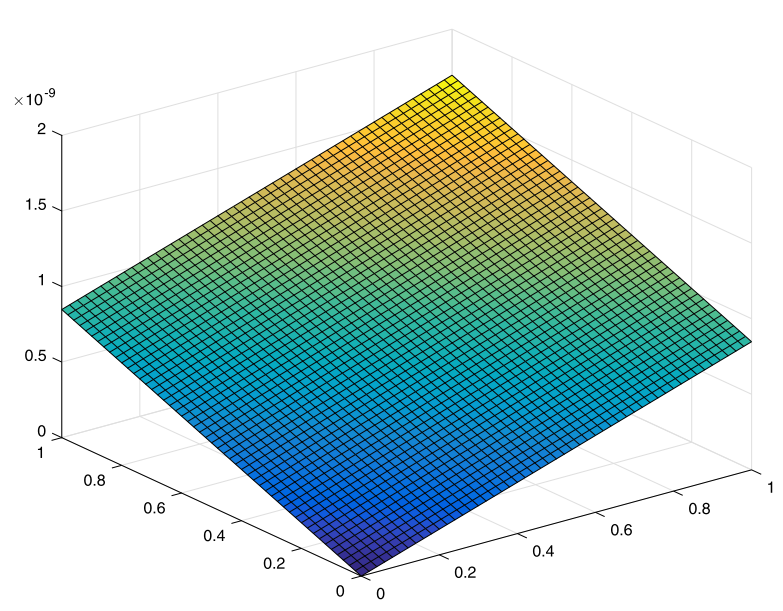

(b) $\bar{e}_{N M}(\mathrm{x}, \mathrm{y}, 0.5)$

Figure 7 Numerical errors with $N=10$ of Example 5.3

Example 5.3 Consider the following 2D-FFIE in Eq. (10):

$$
f_{-}(x, y, r)=(2 r \cos (1-r)-1)\left(1+x^{2}+y-\frac{13}{24}(x+y)\right),
$$




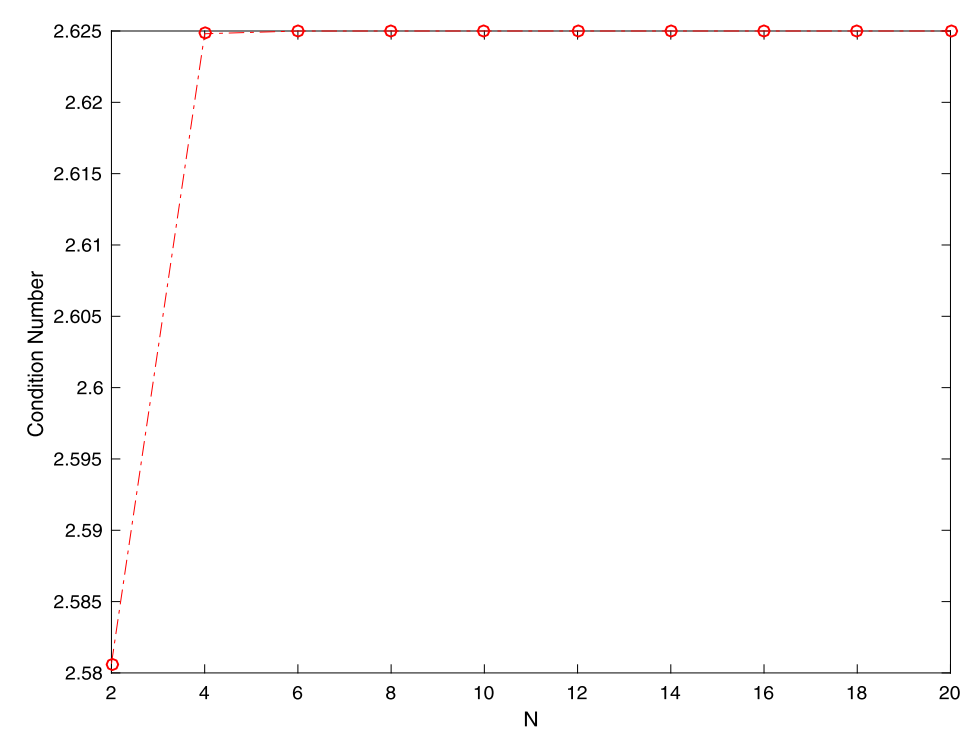

Figure 8 Condition number of the matrix $S-T$ with Example 5.3

$$
\bar{f}(x, y, r)=\left(2-\sin \frac{r \pi}{2}\right)\left(1+x^{2}+y-\frac{13}{24}(x+y)\right)
$$

$\lambda=1$ and $K(x, y, s, t)=(x+y) s t$ for $0 \leq x, y, s, t \leq 1$. The exact solution of this example is

$$
\underline{u}(x, y, r)=(2 r \cos (1-r)-1)\left(x^{2}+y+1\right), \quad \bar{u}(x, y, r)=\left(2-\sin \frac{r \pi}{2}\right)\left(x^{2}+y+1\right) .
$$

The results of Table 5 and Fig. 7 confirm the theoretical results. Figure 8 shows that the condition number of the matrix $S-T$ is bounded. Figure 9 shows the numerical and exact solutions for some values of $x, y$, and $r$. We applied the proposed method to Example 5.3 and compared to the best results obtained in $[41,42]$ in Table 6. From Table 5 we see that our method needs a very small number of collocation points to achieve high accuracy in comparison with the iterative method [41] and the Bernstein polynomials method [42]. Furthermore, the proposed method is easy to implement, in contrast to the iterative method [41], which needs complicated iterations costing too much time.

\section{Conclusion}

In this paper, we introduce a numerical scheme based on the sinc method together with DE transformation to solve 2DFFIE. By solving dual fuzzy linear systems, we obtain approximate solutions. Moreover, we give an error analysis of the proposed method. We provide numerical results to illustrate the effectiveness and accuracy of the presented method. We also compared the proposed scheme to the other numerical methods, which confirms its superiority and the importance of employing the sinc collocation method. In future work, we will study the stability of the current method and utilize the proposed method to deal with other kinds of fuzzy integral equations. 


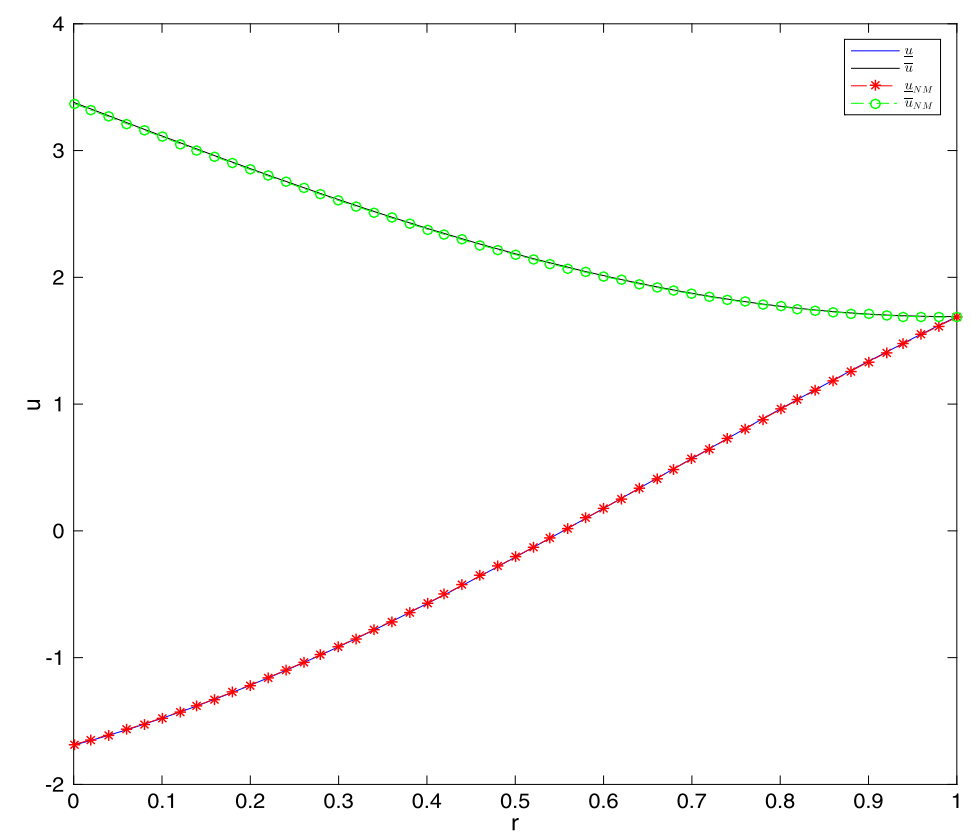

Figure 9 Exact solution and approximate solution with $N=2$ of Example 5.3

Table 6 Comparison of methods with $(x, y)=(0.5 .0 .5)$ of Example 5.3

\begin{tabular}{llll}
\hline$r$ & $\begin{array}{l}\text { Present method } \\
n=2 N+1=17\end{array}$ & $\begin{array}{l}\text { Method of [41] } \\
n=2 N=20 \\
\left(\underline{E}^{r}, \bar{E}^{r}\right)\end{array}$ & $\begin{array}{l}\text { Method of [42] } \\
n=20 \\
\left(\underline{E}^{r}, \bar{E}^{r}\right)\end{array}$ \\
\hline 0.0 & $(3.0 \mathrm{e}-08,5.9 \mathrm{e}-08)$ & $(4.7 \mathrm{e}-05,2.8 \mathrm{e}-05)$ & $(1.0 \mathrm{e}-03,2.2 \mathrm{e}-03)$ \\
0.2 & $(2.1 \mathrm{e}-08,5.0 \mathrm{e}-08)$ & $(3.9 \mathrm{e}-05,8.6 \mathrm{e}-05)$ & $(7.8 \mathrm{e}-04,6.4 \mathrm{e}-03)$ \\
0.4 & $(1.0 \mathrm{e}-08,4.2 \mathrm{e}-08)$ & $(9.0 \mathrm{e}-06,8.0 \mathrm{e}-06)$ & $(1.3 \mathrm{e}-04,8.1 \mathrm{e}-03)$ \\
0.6 & $(3.1 \mathrm{e}-09,3.5 \mathrm{e}-08)$ & $(4.0 \mathrm{e}-06,5.0 \mathrm{e}-06)$ & $(6.1 \mathrm{e}-04,7.3 \mathrm{e}-03)$ \\
0.8 & $(1.7 \mathrm{e}-08,3.1 \mathrm{e}-08)$ & $(1.2 \mathrm{e}-05,3.3 \mathrm{e}-05)$ & $(1.8 \mathrm{e}-03,4.7 \mathrm{e}-03)$ \\
\hline
\end{tabular}

\section{Acknowledgements}

The authors are very grateful to the referees for their detailed comments and valuable suggestions, which greatly improved the manuscript.

\section{Funding}

This work was supported by Key University Science Research Project of Anhui Province (Nos. KJ2019A0786, KJ2019A0782), Ph.D. Research Start-Up Foundation of Anhui Jianzhu University (No. 2018QD36), the programs of Chengdu Normal University (Nos. YJRC2018-1, CS18ZDZ02), and Anhui Provincial Natural Science Foundation (No. 2008085QA12).

\section{Availability of data and materials}

The data used to support the findings of this study are available from the corresponding author upon request.

\section{Competing interests}

The authors declare that they have no competing interests.

\section{Authors' contributions}

All authors contributed equally and read and approved the final version of the manuscript.

\section{Author details}

${ }^{1}$ School of Mathematics and Physics, Anhui Jianzhu University, Hefei, P.R. China. ${ }^{2}$ School of Mathematics, Chengdu Normal University, Chengdu, P.R. China.

\section{Publisher's Note}

Springer Nature remains neutral with regard to jurisdictional claims in published maps and institutional affiliations.

Received: 19 January 2020 Accepted: 24 May 2020 Published online: 15 June 2020 


\section{References}

1. Wu, G.-C., Deng, Z.-G., Baleanu, D., Zeng, D.-Q.: New variable-order fractional chaotic systems for fast image encryption. Chaos, Interdiscip. J. Nonlinear Sci. 29(8), Article ID 083103 (2019)

2. Shiri, B., Baleanu, D.: Numerical solution of some fractional dynamical systems in medicine involving non-singular kernel with vector order. Results Nonlinear Anal. 2(4), 160-168 (2019)

3. Baleanu, D., Shiri, B., Srivastava, H.M., Al Qurashi, M.: A Chebyshev spectral method based on operational matrix for fractional differential equations involving non-singular Mittag-Leffler kernel. Adv. Differ. Equ. 2018(1), Article ID 353 (2018)

4. Shiri, B., Baleanu, D.: System of fractional differential algebraic equations with applications. Chaos Solitons Fractals $120,203-212(2019)$

5. Dadkhah, E., Shiri, B., Ghaffarzadeh, H., Baleanu, D.: Visco-elastic dampers in structural buildings and numerical solution with spline collocation methods. J. Appl. Math. Comput. 63, 29-57 (2019)

6. Mordeson, J., Newman, W.: Fuzzy integral equations. Inf. Sci. 87(4), 215-229 (1995)

7. Friedman, M., Ma, M., Kandel, A.: Numerical solutions of fuzzy differential and integral equations. Fuzzy Sets Syst. 106(1), 35-48 (1999)

8. Park, J.Y., Han, H.K.: Existence and uniqueness theorem for a solution of fuzzy Volterra integral equations. Fuzzy Sets Syst. 105(3), 481-488 (1999)

9. Park, J.Y., Jeong, J.U.: On the existence and uniqueness of solutions of fuzzy Volterra-Fredholm integral equations. Fuzzy Sets Syst. 115(3), 425-431 (2000)

10. Alijani, Z., Baleanu, D., Shiri, B., Wu, G.-C.: Spline collocation methods for systems of fuzzy fractional differential equations. Chaos Solitons Fractals 131, Article ID 109510 (2020)

11. Salahshour, S., Ahmadian, A., Salimi, M., Ferrara, M., Baleanu, D.: Asymptotic solutions of fractional interval differential equations with nonsingular kernel derivative. Chaos, Interdiscip. J. Nonlinear Sci. 29(8), Article ID 083110 (2019)

12. Ahmadian, A., Salahshour, S., Chan, C.S., Baleanu, D.: Numerical solutions of fuzzy differential equations by an efficient Runge-Kutta method with generalized differentiability. Fuzzy Sets Syst. 331, 47-67 (2018)

13. Ahmadian, A., Chan, C.S., Salahshour, S., Vaitheeswaran, V.: FTFBE: a numerical approximation for fuzzy time-fractional Bloch equation. In: 2014 IEEE International Conference on Fuzzy Systems (FUZZ-IEEE), pp. 418-423 (2014)

14. Ahmadian, A., Salahshour, S., Chan, C.S.: A Runge-Kutta method with reduced number of function evaluations to solve hybrid fuzzy differential equations. Soft Comput. 19(4), 1051-1062 (2015)

15. Ahmadian, A., Ismail, F., Salahshour, S., Baleanu, D., Ghaemi, F.: Uncertain viscoelastic models with fractional order: a new spectral tau method to study the numerical simulations of the solution. Commun. Nonlinear Sci. Numer. Simul. 53, 44-64 (2017)

16. Babolian, E., Goghary, H.S., Abbasbandy, S.: Numerical solution of linear Fredholm fuzzy integral equations of the second kind by Adomian method. Appl. Math. Comput. 161(3), 733-744 (2005)

17. Abbasbandy, S., Babolian, E., Alavi, M.: Numerical method for solving linear Fredholm fuzzy integral equations of the second kind. Chaos Solitons Fractals 31(1), 138-146 (2007)

18. Ziari, S., Ezzati, R., Abbasbandy, S.: Numerical solution of linear fuzzy Fredholm integral equations of the second kind using fuzzy Haar wavelet. In: Advances in Computational Intelligence, pp. 79-89. Springer, Berlin (2012)

19. Bica, A.M., Popescu, C.: Numerical solutions of the nonlinear fuzzy Hammerstein-Volterra delay integral equations. Inf. Sci. 223, 236-255 (2013)

20. Araghi, M.F., Parandin, N.: Numerical solution of fuzzy Fredholm integral equations by the Lagrange interpolation based on the extension principle. Soft Comput. 15(12), 2449-2456 (2011)

21. Jafarzadeh, Y.: Numerical solution for fuzzy Fredholm integral equations with upper-bound on error by splines interpolation. Fuzzy Inf. Eng. 4(3), 339-347 (2012)

22. Araghi, M.F., Gelian, G.K.: Solving fuzzy Fredholm linear integral equations using Sinc method and double exponential transformation. Soft Comput. 19(4), 1063-1070 (2015)

23. Baghmisheh, M., Ezzati, R.: Numerical solution of nonlinear fuzzy Fredholm integral equations of the second kind using hybrid of block-pulse functions and Taylor series. Adv. Differ. Equ. 2015(1), Article ID 51 (2015)

24. Rivaz, A., Yousefi, F.: Modified homotopy perturbation method for sloving two-dimensional fuzzy Fredholm integral equations. Int. J. Appl. Math. 25(4), 591-602 (2012)

25. Ezzati, R., Ziari, S.: Numerical solution of two-dimensional fuzzy Fredholm integral equations of the second kind using fuzzy bivariate Bernstein polynomials. Int. J. Fuzzy Syst. 15(1), 84-89 (2013)

26. Rivaz, A., Yousefi, F., Salehinejad, H.: Using block pulse functions for solving two-dimensional fuzzy Fredholm integral equations of the second kind. Int. J. Appl. Math. 25(4), 571-582 (2012)

27. Mirzaee, F., Yari, M.K., Hadadiyan, E.: Numerical solution of two-dimensional fuzzy Fredholm integral equations of the second kind using triangular functions. Beni-Suef Univ. J. Basic Appl. Sci. 4, 109-118 (2015)

28. Bica, A.M., Popescu, C.: Fuzzy trapezoidal cubature rule and application to two-dimensional fuzzy Fredholm integral equations. Soft Comput. 21, 1229-1243 (2015)

29. Sadatrasoul, S.M., Ezzati, R.: Iterative method for numerical solution of two-dimensional nonlinear fuzzy integral equations. Fuzzy Sets Syst. 280, 91-106 (2014)

30. Sadatrasoul, S.M., Ezzati, R.: Numerical solution of two-dimensional nonlinear Hammerstein fuzzy integral equations based on optimal fuzzy quadrature formula. J. Comput. Appl. Math. 292, 430-446 (2016)

31. Goetschel, R., Voxman, W.: Elementary fuzzy calculus. Fuzzy Sets Syst. 18(1), 31-43 (1986)

32. Wu, C., Gong, Z.: On Henstock integral of fuzzy-number-valued functions (I). Fuzzy Sets Syst. 120(3), 523-532 (2001)

33. Gal, S.G.: Approximation theory in fuzzy setting (2000)

34. Anastassiou, G.A.: Fuzzy Mathematics: Approximation Theory. Studies in Fuzziness and Soft Computing, vol. 251. Springer, Berlin (2010)

35. Wu, H.-C.: The improper fuzzy Riemann integral and its numerical integration. Inf. Sci. 111(1), 109-137 (1998)

36. Ma, M., Friedman, M., Kandel, A.: Duality in fuzzy linear systems. Fuzzy Sets Syst. 109(1), 55-58 (2000)

37. Muhammad, M., Nurmuhammad, A., Mori, M., Sugihara, M.: Numerical solution of integral equations by means of the Sinc collocation method based on the double exponential transformation. J. Comput. Appl. Math. 177(2), 269-286 (2005) 
38. Mori, M., Sugihara, M.: The double-exponential transformation in numerical analysis. J. Comput. Appl. Math. 127(1), 287-296 (2001)

39. Yazdi, M.H., Gelian, G.K., et al.: Error estimate in the sinc collocation method for Volterra-Fredholm integral equations based on DE transformation. Electron. Trans. Numer. Anal. 30, 75-87 (2008)

40. Khastan, A., Ivaz, K.: Numerical solution of fuzzy differential equations by Nyström method. Chaos Solitons Fractals 41(2), 859-868 (2009)

41. Sadatrasoul, S.M., Ezzati, R.: Quadrature rules and iterative method for numerical solution of two-dimensional fuzzy integral equations. Abstr. Appl. Anal. 2014, Article ID 413570 (2014)

42. Ezzati, R., Sadatrasoul, S.M.: Application of bivariate fuzzy Bernstein polynomials to solve two-dimensional fuzzy integral equations. Soft Comput. 21(14), 3879-3889 (2017)

Submit your manuscript to a SpringerOpen ${ }^{\circ}$ journal and benefit from:

- Convenient online submission

- Rigorous peer review

- Open access: articles freely available online

- High visibility within the field

- Retaining the copyright to your article

Submit your next manuscript at $\boldsymbol{\nabla}$ springeropen.com 